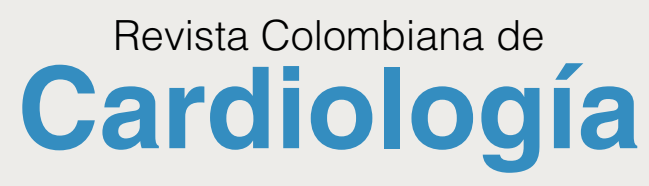

www.elsevier.es/revcolcar

CARTAS AL EDITOR

\section{Parada cardiaca debido a hemorragia subaracnoidea simulando un síndrome coronario agudo}

\section{Cardiac arrest due to a subarachnoid hemorrhage simulating an acute coronary syndrome}

\section{Sr. Editor:}

El síndrome coronario agudo es la principal causa de parada cardiaca extrahospitalaria en países occidentales ${ }^{1}$. Las guías europeas de reanimación cardio-pulmonar recomiendan una coronariografía urgente con miras a realizar una intervención coronaria percutánea primaria en los supervivientes de una parada cardiaca extrahospitalaria ${ }^{2,3}$. Las causas extracardiacas de parada cardiaca extrahospitalaria como la hemorragia subaracnoidea son menos comunes y de mal pronóstico.

Se presentan dos casos de parada cardiaca extrahospitalaria por hemorragia subaracnoidea que simularon un síndrome coronario agudo, el cual requirió coronariografía urgente.

\section{Caso 1}

Mujer de 47 años de edad, sin antecedentes patológicos de interés, quien mientras realizaba actividad física tuvo una parada extrahospitalaria, sin dolor torácico previo. Se iniciaron maniobras de reanimación cardio-pulmonar básica y avanzada, con un primer ritmo de asistolia. El electrocardiograma (ECG) inicial posresucitación mostró ritmo sinusal, con descenso difuso del segmento ST y elevación del segmento ST en la derivación AVR, que sugería enfermedad multivaso o del tronco común de la coronaria izquierda (fig. $1 \mathrm{~A})$. Se administró aspirina endovenosa, ticagrelor $180 \mathrm{mg}$ por sonda nasogástrica y 5.000 unidades de heparina sódica. Se activó código infarto con traslado urgente a sala de hemodinámica. La coronariografía mostró arterias coronarias lisas sin lesiones (fig. $1 \mathrm{~B}$ ), en tanto que la analítica elevación de troponina । hasta $12,1 \mathrm{ng} / \mathrm{dl}$. Debido al cuadro que se presentó como síncope brusco, con un primer ritmo de asistolia y pupilas midriáticas, se realizó tomografía computarizada (TC) de cráneo en la que se evidenció hemorragia subaracnoidea extensa en cisternas de la base y difusa en ambos hemisferios cerebrales, además de contaminación intraventricular supra e infratentorial, con edema cerebral difuso (fig. 1 C), e imposibilidad para valorar malformaciones vasculares. Se desestimó para tratamiento quirúrgico dada la extensión de la hemorragia subaracnoidea. Se diagnosticó muerte encefálica luego de 12 horas del evento.

\section{Caso 2}

Varón de 49 años, sin antecedentes de interés, quien presentó una parada cardiaca extrahospitalaria mientras trabajaba, sin dolor torácico previo, con primer ritmo de asistolia. En el ECG inicial posresucitación se observó elevación del segmento ST de v1-v6 que sugería un infarto agudo de miocardio anterior. Se activó código infarto y se administró Aspirina vía endovenosa más 5.000 unidades de heparina sódica. Se realizó coronariografía urgente en la que se observaron arterias coronarias sin lesiones. En ecocardiograma se evidenció acinesia de segmentos apicales y pico de troponina । de 5,4ng/dl, que inicialmente sugirió un cuadro de miocardiopatía de estrés o síndrome de takotsubo. Ante la persistencia de Glasgow de 3 sin sedación, se realizó TC de cráneo que objetivó hemorragia subaracnoidea masiva con edema cerebral, correspondiente a hemorragia subaracnoidea grado 4 de la escala de Fisher por rotura de aneurisma de la arteria vertebral derecha. El tratamiento quirúrgico fue desestimado debido a la extensión de la hemorragia subaracnoidea. Se diagnosticó muerte encefálica pasadas 24 horas del evento.

Los hallazgos en el electrocardiograma y en el ecocardiograma en el contexto de una hemorragia subaracnoidea pueden imitar los cambios causados por síndromes coronarios agudos y por lo tanto dar lugar a un retraso en el tratamiento de la enfermedad primaria ${ }^{4}$.

El mecanismo probable propuesto para este evento es que la hemorragia subaracnoidea induce una descarga de catecolaminas excesiva, que produce aturdimiento miocárdico por estimulación simpática, disfunción cardiaca, elevación de enzimas cardiacas y cambios en el electrocardiograma ${ }^{5}$.

En pacientes inconscientes es difícil el diagnóstico diferencial de un síndrome coronario agudo. En los casos expuestos el electrocardiograma sugirió enfermedad coronaria importante, por lo que se administraron antiplaquetarios y anticoagulantes que pudieron empeorar aún más el pronóstico de la hemorragia subaracnoidea.

Valorar una TC de cráneo previo a la activación de un código infarto podría ser el método más efectivo para la identificación rápida de una hemorragia subaracnoidea en 

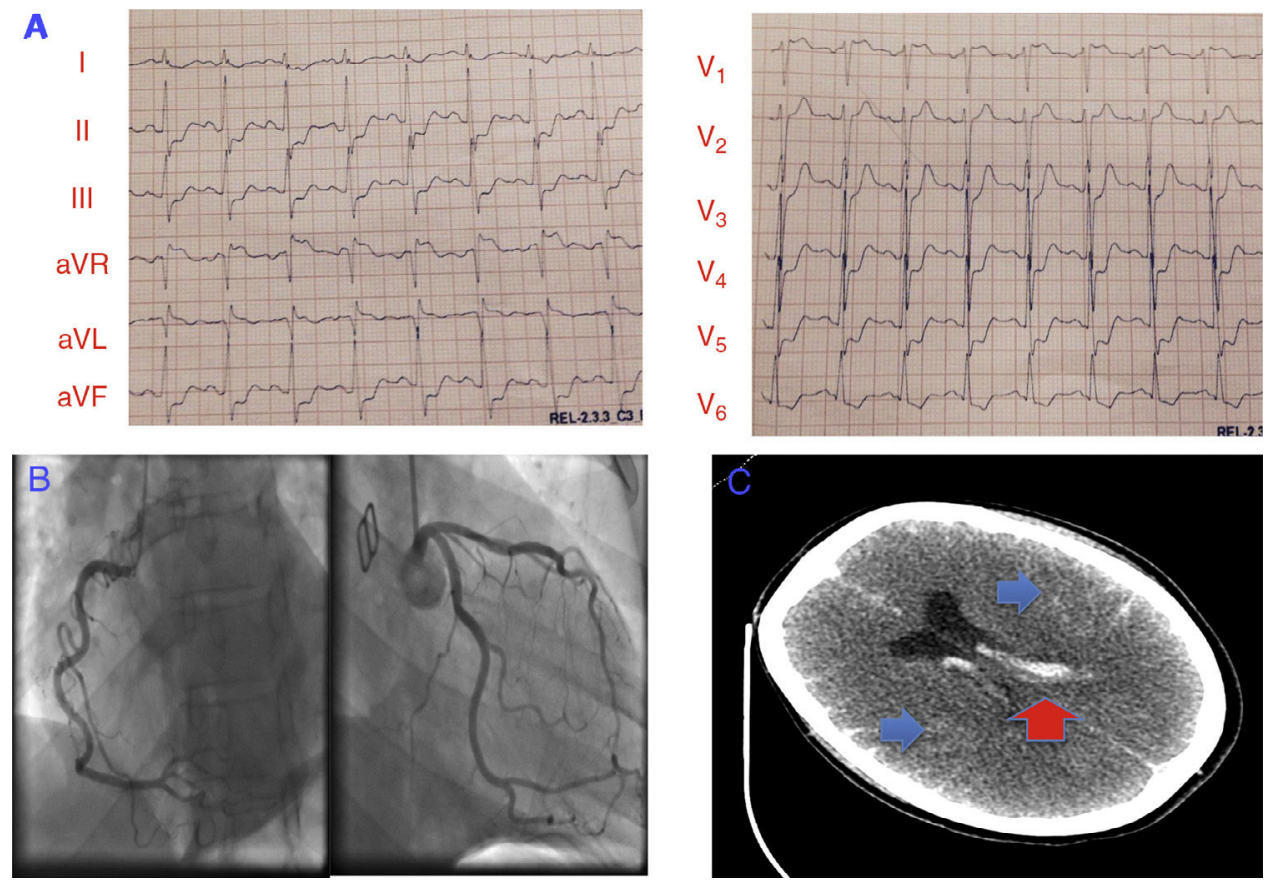

Figura 1

este contexto, sobre todo en pacientes jóvenes sin factores de riesgo cardiovascular, sin dolor torácico previo y con un primer ritmo de asistolia, como ocurrió en estos casos.

\section{Conflicto de intereses}

Los autores declaran no tener ningún conflicto de intereses.

\section{Bibliografía}

1. Dumas F, Cariou A, Manzo-Silberman S, Grimaldi D, Vivien B, Rosencher J, et al. Immediate percutaneous coronary intervention is associated with better survival after out-of-hospital cardiac arrest: insights from the PROCAT (Parisian Region Out of hospital Cardiac Arrest) registry. Circ Cardiovasc Interv. 2010;3:200-7.

2. Nikolau N, Arntz HR, Bellou A, Beygui F, Bossaert LL, Cariou A. European resuscitation council guidelines for resuscitation 2015 section 5. Management of acute coronary syndrome. Resuscitation. 2015;95:264-77.

3. Windecker S, Kolh P, Alfonso F, Collet JP, Cremer J, Falk V, et al. 2014 ESC/EACTS Guidelines on myocardial revascularization: the task force on myocardial revascularization of the European Society of Cardiology (ESC) and the European Association for CardioThoracic Surgery (EACTS) developed with the special contribution of the European Association of Percutaneous Cardiovascular Interventions (EAPCI). Eur Heart J. 2014;35: 2541-619.
4. Yamashina $Y$, Yagi T, Ishida A, Mibiki Y, Sato H, Nakagawa $\mathrm{T}$, et al. Differentiating between comatose patients resuscitated from acute coronary syndrome-associated and subarachnoid hemorrhage-associated out-of-hospital cardiac arrest. J Cardiology. 2015;65:508-13.

5. Lee V, Oh J, Sharon L, Wijdicks EF. Mechanisms in neurogenic stress cardiomyopathy after aneurysmal subarachnoid hemorrhage. Neurocrit Care. 2006;5:243-9.

\section{Gustavo Jiménez-Brítez*, Paula Sánchez-Somonte y Xavier Freixa}

Departamento de Cardiología, Hospital Clínic de Barcelona, Institut d'Investigacions Biomèdiques August Pi i Sunyer (IDIBAPS), Barcelona, España

* Autor para correspondencia.

Correo electrónico: gujimene@clinic.ub.es (G. Jiménez-Brítez).

http://dx.doi.org/10.1016/j.rccar.2016.10.041 0120-5633/

(C) 2016 Sociedad Colombiana de Cardiología y Cirugía Cardiovascular. Publicado por Elsevier España, S.L.U. Este es un artículo Open Access bajo la licencia CC BY-NC-ND (http: / / creativecommons.org/licenses/by-nc-nd/4.0/). 